Historic, Archive Document

Do not assume content reflects current scientific knowledge, policies, or practices. 


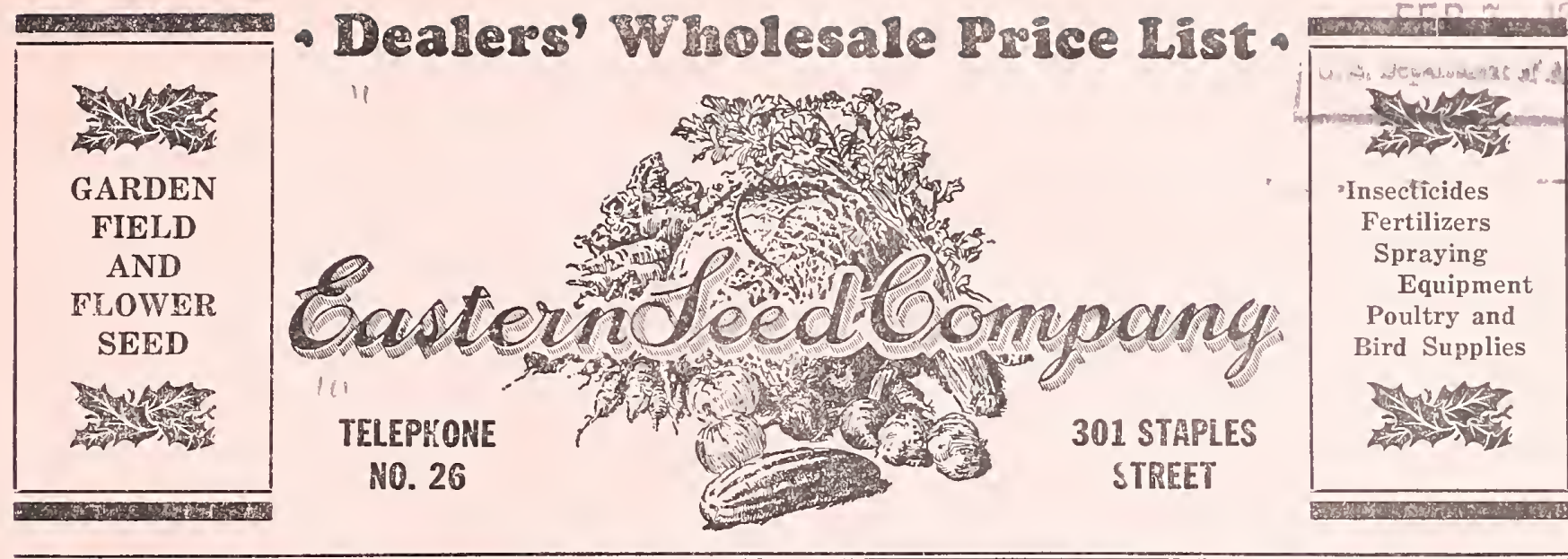

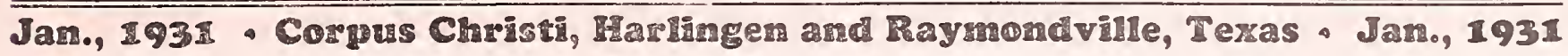

\section{NON WARRANTYY}

WE QUOTE TODAY'S VALUES SUBJECT TO MARKET FLUCTUATION F.O.B. OUR STORES

NOTICE-Eastern Seed Company does not give, and their agents and employees are forbidden to give, any. warranty, express or implied, as to description, quality, productiveness or any other matter of any seeds, bulbs or plants we sell and will not be in any way responsible for the crop. All sales subject to loss or damage by fire, shortage or failure of crops, and other contingencies beyond our control. Prices subject to change without notice.

Purity and germination tests are made for guidance only and are without guarantee.

\section{GARDIEN AND EIELD STED}

Our stocks of fall garden seed are arriving now, and while some varieties of cabbage and onions are going to be short, we feel that we are in a position to take care of any normal demand.

We anticipate higher prices as the planting season advances and believe that it would be a good idea for those who intend to handle some seed to book their orders now.

Our stocks of seed come from the best known growers in this country and Europe, and this, with our modern method of testing for germination, places us in a position to supply you with seed which will grow, run true to variety and are best adapted to this section of the state. We are also booking orders now for spring delivery. No doubt our salesman will call on you in the near future and quote you prices, but in case he does not we would be glad if you would send us a list of the items in which you are interested and we will quote you by return mail.

We are trying to list the standard staple items that are planted in this telritory and you may need other items that we do not list, so if you will send us a list of these we will be glad to quote you prices.

The prices quoted in this list are F.O.B. Our Stores, in less than carload lots and freight prepaid in carload lots.

\section{SELECT TESTED SEED}

\section{BEANS}

Dwarf Varieties-

Early Red Valentine

Black Valentine

Burpee's Stringless

Giant Stringless

Wax Pod Varieties-

Pencil Pod Black Wax

Golden Wax

\section{BUSH LIMAS}

Henderson Bush

Old Fashioned Speckled

POLE LIMAS

Small Sieva

Speckled Pole

$$
\text { le }
$$

\section{BEETS}

Crosby's Egyptian

Detroit Dark Red

Mammoth Long Red Stock
$10 \mathrm{lbs} .50 \mathrm{lbs} . \quad 100 \mathrm{lbs}$.

\section{$\begin{array}{lll}1.80 & 8.50 & 16.00\end{array}$}

$\begin{array}{lll}1.80 & 8.50 & 16.00\end{array}$

$\begin{array}{lll}1.80 & 8.50 & 16.00\end{array}$

$\begin{array}{lll}1.80 & 8.50 & 16.00\end{array}$

$\begin{array}{lll}2.00 & 9.00 & 17.00\end{array}$

$2.25 \quad 9.00$

$\begin{array}{ll}2.50 & 9.00\end{array}$

$\begin{array}{ll}2.25 & 10.00\end{array}$

$2.50 \quad 11.00$

$\begin{array}{lll}4.50 & 20.00 & 35.00\end{array}$

$\begin{array}{lll}4.50 & 20.00 & 35.00\end{array}$

$\begin{array}{lll}4.00 & 18.00 & 35.00\end{array}$

CABBAGE

All Head Early

Jersey Wakefield

Charleston Wakefield

Coperihagen Market

Glory of Enkhuizen

Stein's Early Flat Dutch

Clark's Medium Early Flat HeadCANTALOUPES

Hearts of Gold Select.

Honey Ball

Our Special, orange flesh

Pollock 10-25

Rockyford, select

\section{CARROTS}

Improved Chantenay

Oxheart

Danish Grown-

Early Snowball

\section{CAULIFLOWER} per lb. $\quad 12.00$
$10 \mathrm{lbs} . \quad 50 \mathrm{lbs} . \quad 100 \mathrm{lbs}$.

$\begin{array}{lll}12.50 & 60.00 & 115.00\end{array}$

$\begin{array}{lll}10.00 & 47.50 & \mathbf{9 0 . 0 0}\end{array}$

$\begin{array}{lll}10.00 & 47.50 & 90.00\end{array}$

$\begin{array}{llr}17.00 & 82.50 & 160.00\end{array}$

$\begin{array}{lll}14.00 & 67.50 & 125.00\end{array}$

$\begin{array}{lll}11.00 & 52.50 & 100.00\end{array}$

$\begin{array}{lll}14.00 & 67.50 & 125.00\end{array}$

$\begin{array}{lll}8.00 & 37.50 & 70.00\end{array}$

$\begin{array}{rrr}17.50 & 85.00 & 165.00\end{array}$

$\begin{array}{rrr}8.50 & 40.00 & 75.00\end{array}$

$\begin{array}{lll}7.50 & 35.00 & 65.00\end{array}$

$\begin{array}{lll}7.50 & 35.00 & 65.00\end{array}$

$\begin{array}{lll}5.50 & 25.00 & 45.00\end{array}$ $\begin{array}{lll}5.50 & 25.00 & 45.00\end{array}$

All Prices F.O.B. Our Stores, Bags Extra at Cost 


\section{CELERY}

Golden Self Blanching -...--.-.-.-per lb. 2.50

\section{COLLARDS}

Georgia or Southern

\section{CUCUMBERS}

Rice Early Fortune

Esco Brand Early Fortune

Chicago Pickling

\section{EGG PLANT}

Black Beauty

$1 \mathrm{lb} . \quad 10 \mathrm{lbs} .100 \mathrm{lbs}$.

$3.50 \quad 32.50$

KALE

Dwarf Green Curled.

KOHL RABI

Early White Vienna

$1.10 \quad 10.00$

\section{LETTUCE}

Big Boston

Iceberg

New York or Wonderful

$.75 \quad 7.00$

$1.00 \quad 9.00$

$1.50 \quad 14.00$

\section{MANGEL WURZELS}

Mammonth Long Red

$\begin{array}{lll}.45 & 4.00 \quad 35.00\end{array}$

\section{MUSTARD}

Chinese Giant, smooth leaf

Southern Giant, curled

$\begin{array}{lll}.40 & 3.50 & 30.00\end{array}$

$\begin{array}{lll}.40 & 3.50 & 30.00\end{array}$

OKRA

White Velvet

$\begin{array}{lll}3.5 & 3.00 & 25.00\end{array}$

Long Green

$\begin{array}{lll}.35 & 3.00 & 25.00\end{array}$

\section{ONION}

Yellow Bermuda

Crystal Wax

Onion Plants

2.3522 .50 Ash $2.85 \quad 2.75$ Ask Ask for Prices

\section{ONION SETS}

White

Yellow

Ask for Prices

Ask for Prices

\section{PARSLEY}

Double Curled

\section{PARSNIP}

Hollow crown or Sugar

$.40 \quad 3.50$

\section{PEAS}

Semi Dwarf Varieties-

First and Best

Thos. Laxton

\section{PEPPER}

Chinese Giant

$1 \mathrm{lb} . \quad 10 \mathrm{lbs} .100 \mathrm{lbs}$.

Ruby King

$\begin{array}{ll}4.50 & 40.00\end{array}$

Worldbeater

$2.50 \quad 22.50$

Long Red Cayenne

$2.75 \quad 25.00$

Small Red Chili.

$\begin{array}{ll}2.75 & 25.00\end{array}$

$3.75 \quad 35.00$

\section{IRISH POTATOES}

Irish Cobblers, two bu. bags

3.25 Cwt.

Triumphs, two bu. bags

\section{PUMPKIN}

$\begin{array}{lll}\text { Green Striped Cushaw } & \mathbf{7 5} & \mathbf{6 . 5 0} \\ \text { Japanese Pie } & \mathbf{. 7 5} & \mathbf{7 . 0 0} \\ \text { Kentucky Field } & \mathbf{. 5 0} & \mathbf{4 . 5 0} \\ \text { Mammoth Tours } & .85 & 8.00 \\ \text { Small Sugar } & \mathbf{. 7 5} & \mathbf{7 . 0 0}\end{array}$

\section{RADISH}

Early Scarlet Turnip

White Tip

Scarlet Globe

Long Red

White Icicle

SPINACH

Bloomsdale, Re-selected

\section{SQUASH}

Early White Bush

Yellow Summer or Crook Neck ......

Hubbard

Cocozelle

\subsection{0}

40.00

$\begin{array}{lll}.50 & 4.50 & 40.00\end{array}$

$\begin{array}{lll}50 & 4.50 & 40.00\end{array}$

$\begin{array}{lll}.50 & 4.50 & 40.00\end{array}$

$50 \quad 4.50$

40.00

.60

5.50

50.00

$\begin{array}{lll}.60 & 5.50 & 50.00\end{array}$

$\begin{array}{lll}.75 & 7.00 & 65.00\end{array}$

$\begin{array}{lll}1.00 & 10.00 & 95.00\end{array}$

\section{TOMATO}

Cooper Special

Dwarf Champion

Gulf State Market

June Pink

$\mathrm{McGee}$

Narglobe

Ponderosa

Yellow Pear

32.50

300.00

$32.50 \quad 300.00$

$32.50 \quad 300.00$

$34.00 \quad 325.00$

\section{TURNIP}

Amber Globe

Pomeranian White Globe

Purple Top White Globe

Purple Top Strap Leaf

Purple Top Rutabega

White Egg

Shogoin

WATERMELON

Halbert Honey, Select

Irish Gray, Select

Kleckley Sweet, Select

New Wonder

Stone Mountain

Tom Watson

Tom Watson, Weatherford Select....

$\begin{array}{lll}.35 & \mathbf{3 . 2 0} & \mathbf{3 0 . 0 0} \\ .35 & \mathbf{3 . 2 0} & \mathbf{3 0 . 0 0} \\ .35 & \mathbf{3 . 2 0} & \mathbf{3 0 . 0 0} \\ .35 & \mathbf{3 . 2 0} & \mathbf{3 0 . 0 0} \\ .35 & \mathbf{3 . 2 0} & \mathbf{3 0 . 0 0} \\ .35 & \mathbf{3 . 2 0} & \mathbf{3 0 . 0 0} \\ .65 & 6.00 & \mathbf{5 5 . 0 0}\end{array}$




\section{SELECTED FIELD SEED}

(ALL TESTED AND TAGGED IN ACCORDANCE WITH STATE LAWS)

\section{ALFALFA}

Hairy Peruvian

\section{BARLEY}

Bearded Winter

\section{CANE SEED}

Extra Fancy Red Top

Fancy Red Top

Fancy Japanese Honey Drip

\section{POP CORN}

White Rice

Queen's Golden

Japanese Hulless

\section{SEED CORN}

Surecropper

Mexican June

Indian Squaw

Southern Yellow Dent.

White Pearl

Stowell's Evergreen

Golden Bantam

\section{CLOVERS}

White Flowering Sweet

California Burr

\section{COTTON SEED}

Bennett's New High Speed

Mississippi Delfos

Mississippi Stoneville -_-_._-

10 Bu Lots Sack Lots

$2.50 \quad 2.60$ per bu.

2.502 .60 per bu.

$2.50 \quad 2.60$ per bu.

27.502 .75 per bu.

$1 \mathrm{lb} .10 \mathrm{lbs} .100 \mathrm{lbs}$.

$\begin{array}{lll}.20 & 1.90 & 18.00\end{array}$

$\begin{array}{lll}.20 & 1.90 & 18.00\end{array}$

We can also supply all standard varieties of second year seed grown in Nueces and San Patricio Counties.

\section{DARSO}

Fancy

$4.50 \mathrm{Cwt}$.

EGYPTIAN WHEAT

Fancy

$5.00 \mathrm{Cwt}$

\section{FETERITA}

Fancy

$4.50 \mathrm{Cwt}$.
1.20

Ask for Prices

Ask for Prices

2.60 per bu.

1.25 per bu.

2.00 per bu.

2.25 per bu.

\section{an}

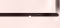

Ask for Prices

COW PEAS

Whippoorwill

Blackeye

Brown Crowder

White Cream

\section{GRASS SEED}

Esco Brand Sudan
Fancy Sudan

Rhodes -.. -..-

Fancy Bermuda

-

$-1-2$

\section{HEGARI}

We are pioneers in the handling of Arizona Hegari in Southwest Texas and have handled millions of pounds during the past few years, and it has given perfect satisfaction. We do not hesitate to recommend it to our trade. It grows to a nice, uniform height and makes fine big heads.

Esco Brand Fancy.

$4.00 \mathrm{Cwt}$.

\section{KAFFIR}

Fancy Black Hull White Okla.

$3.00 \mathrm{Cwt}$.

Black Hull White Texas

$2.60 \mathrm{Cwt}$.

\section{MILO MAIZE}

Esco Brand Double Dwarf Arizona Fine large heads without so much stalk; very early.

Fancy Dwarf Yellow Texas

4.00 Cwt.

$2.60 \mathrm{Cwt}$.

\section{MILLET}

Fancy Big German

Ask for Prices

\section{SEED OATS}

Fancy Texas Red, rust proof

.80

\section{PEANUTS}

Fancy Spanish, hand picked --.Fancy Tennessee Red _................

Ask for Prices Ask for Prices

\section{RYE}

Fancy Black Winter

1.50

\section{SUNFLOWER}

\section{BIRD SUPPLIES}

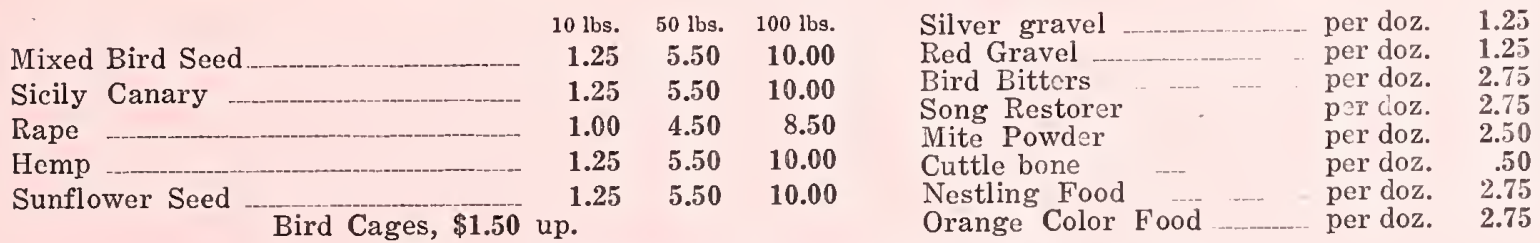




\section{ESCO Brand Select Tested Flower Seed}

Our flower seed are grown for us by the foremost growers in California and we buy only the finest grades, you may therefore expect good flowers when you plant our seed.

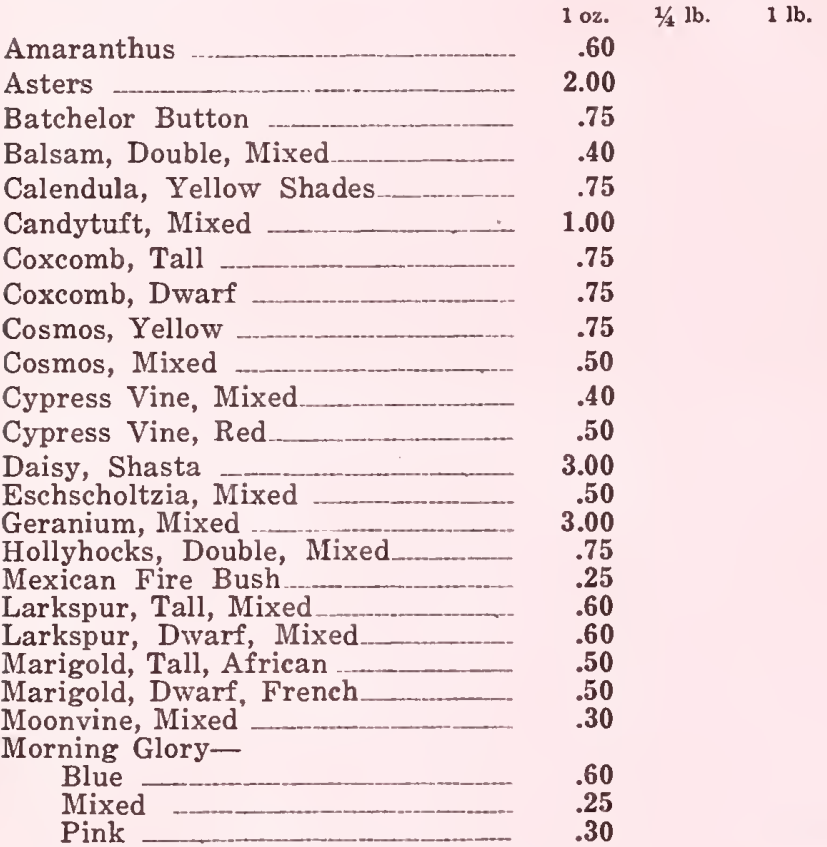

Nasturtiums-

$$
\text { 1 oz. } \quad 1 / 4 \mathrm{lb} . \quad 1 \mathrm{lb} \text {. }
$$

Dwarf

lb.

.75

Tall

.75

Pansy, Mixed

Petunia, Mixed

Phlox, Mixed

Pinks, Double Mixed

Poppy, Double Shirley, Mixed

Poppy, Double Carnation Fl. Mixed

Portulaca, Double Mixed.

Salvia, Scarlet Sage

Snapdragon, Tall, Mixed

Snapdragon, Dwarf, Mixed

Stocks, Fine Mixed

Sweet Peas-Spencer's Early Fl.

$$
\text { Mixed }
$$

Sweet William, Mixed _.____.........

Verbena-

Mixed

Red

Purple

Pink

White

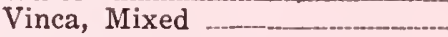

Zinnias--

California Giant, Mixed

California, Miniature, Mixed -

Giant Red

Giant Pink

Giant Purple

Giant Lavender

Giant Orange

Giant Yellow
3.00

.50

$.75 \quad 2.75$

$.50 \quad 1.90$

$.50 \quad 1.90$

$.50 \quad 1.90$

2.50

3.00

$.75 \quad 2.75$

$.75 \quad 2.75$

1.25

10.00

$.20 \quad .75$

3.00

.50

$\begin{array}{rr}.90 & 3.50\end{array}$

$1.00 \quad 3.75$

$1.00 \quad 3.75$

$1.00 \quad 3.75$

$1.00 \quad 3.75$

$.75 \quad 2.75$

$65 \quad 2.55$

$\begin{array}{ll}.50 & 1.90\end{array}$

$.85 \quad 3.25$

$.85 \quad 3.25$

$.85 \quad 3.25$

$.85 \quad 3.25$

.85
$\quad 3.25$

$.85 \quad 3.25$

\section{FERTILIZERS}

Vigoro-

$5 \mathrm{lb}$. Can, 14 to case

$\$ 4.62$

25 lb. Sack

1.15

$50 \mathrm{lb}$. Sack

$100 \mathrm{lb}$. Sack

2.00

3.50

Fidelity 4-8-6, per cwt. 2.50 Per ton_Ask for Prices

Fidelity 4-12-4, per cwt. Per ton Ask for Prices

Fidelity 6-12-6, per cwt._.___._._._Per ton_-_..Ask for Prices

Nitrate of Soda_ Ask for Prices

Acid Phosphate ._._._._Ask for Prices

\section{SPRAYERS}

Hudson Misty, tin

Hudson Misty, brass

Doz. $\$ 4.50$

Hudson Continuous, brass

Doz. 9.00

Hudson Junior, galvanized

Doz. 12.80

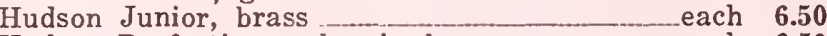

Hudson Perfection, galvanized____each $\mathbf{6 . 5 0}$

Hudson Perfection, brass _each $\mathbf{8 . 5 0}$

Hudson Clipper, galvanized _________........each 4.50

Hudson Barrel

each 5.00

\section{SEED DISINFECTANTS}

Semesan, $1 \mathrm{oz}$. cans, each $\$ \mathbf{. 5 0}$ less Dealers Discount Semesan, 1 lb. cans, each 2.75 _... less Dealers Discount Ceresan, 5 lb. cans, each $3.00 \ldots$ less Dealers Discount Ceresan, $25 \mathrm{lb}$. cans, each 12.50 __ less Dealers Discount

\section{INSECTICIDES}

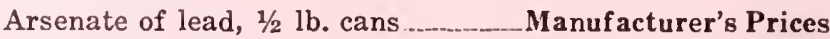
Black leaf, $401 \mathrm{oz}$. Manuf acturer's Prices

Black leaf, $40 \mathrm{l} 1 / 2 \mathrm{lb}$. Manuf acturer's Prices

Black leaf, $402 \mathrm{lb}$. Manufacturer's Prices

Black leaf, $4010 \mathrm{lb}$. Manuf acturer's Prices

Bordeaux Mixture, $1 \mathrm{lb}$. cans Dry Lime Sulphur, 1 lb. cans Case lots at Mfgrs. Price

Red Arrow Spray, 1 oz.

Paris Green, $1 \mathrm{lb}$.

Paris Green, $14 \mathrm{lb}$. Case lots at Mfgrs. Price Case lots at Mfgrs. Price

Paris Green, $100 \mathrm{lb}$.

Paris Green, $250 \mathrm{lb}$.

Pestroy, 1/2 lb.

Pestroy, $1 \mathrm{lb}$

Snarol, $1 \mathrm{lb}$.

Snarol, $3 \mathrm{lb}$.

Cyanogas, $1 / 2 \mathrm{lb}$.

Cyanogas, $1 \mathrm{lb}$.

Cyanogas, $5 \mathrm{lb}$.

London Purple, $1 / 4 \mathrm{lb}$.

London Purple, $1 \mathrm{lb}$.

Pulvex (for fleas)

U-K-E-, $1 / 2$ pint

U-K-E-, 1 pint

U-K-E-, quart Case lots at Mfgrs. Price Manuf acturer's Price Manufacturer's Price Manuf acturer's Price Case lots at Mfgrs. Price Case lots at Mfgrs. Price Case lots at Mfgrs. Price Case lots at Mfgrs. Price Case lots at Mfgrs. Price Case lots at Mfgrs. Price Case lots at Mfgrs. Price Case lots at Mfgrs. Price Case lots at Mfgrs. Price Case lots at Mfgrs. Price Case lots at Mfgrs. Price Case lots at Mfgrs. Price

U-K-E-, gallon Case lots at Mfgrs. Price 